

\title{
Preparing Starchy Foods Containing Silver Nanoparticles and Evaluating Antimicrobial Activitiy
}

\author{
Mohsen Adeli ${ }^{1}$, Hasan Hosainzadegan ${ }^{2, *}$, Iraj Pakzad ${ }^{3}$, Fatemeh Zabihi ${ }^{1}$, Maedee Alizadeh ${ }^{2}$, \\ Fattane Karimi $^{2}$ \\ ${ }^{1}$ Department of Chemistry, Faculty of Science, Lorestan University, Khoramabad, IR Iran \\ ${ }^{2}$ Maragheh Faculty of Nursing and Midwifery, Tabriz University of Medical Sciences, Tabriz, IR Iran \\ ${ }^{3}$ Department of Microbiology and Clinical Microbiology Research Center, Faculty of Medicine, Ilam University of Medical Sciences, Ilam, IR Iran \\ *Corresponding author: Hasan Hosainzadegan, Maragheh Faculty of Nursing \& Midwifery, Tabriz University of Medical Sciences, Tabriz, IR Iran. Tel: +98- \\ 9147667729, Fax:+98-4212276363, E-mail: hasanhosainy122@yahoo.com.
}

\begin{abstract}
A B S T R A C T
Background: Silver has been used from ancient times by humans, and recently nanoparticles of silver have been used in many aspects of human life including as a potent antibacterial agent.

objectives: In the current study a completely green method to prepare silver nanoparticles is reported. Two types of starches (potato and corn starches) were used to produce and stabilize silver nanoparticles. Antimicrobial activities of prepared nanofoods were investigated.

Materials and Methods: Nanosilver particles have been produced using high temperature water containing bulk silver nitrate and starch sources. Presence of nanosilver containing particles and formation of the nanoparticles were confirmed using transmission electron microscopy (TEM) and ultraviolet-visible spectroscopy, respectively. The anti-bacterial activity of emulsions made from nanoparticles containing starches was evaluated by assessing minimum inhibitory concentrations (MIC) against the studied standard, and pathogenic bacteria.

Results: Findings of the current study indicated that the size and shape of silver nanoparticles depended on the type of starch. For example for a special type of potato starch the cubic silver nanoparticles were obtained whereas for other types of potato starch and corn starch spherical silver nanoparticles were produced. Nanoparticle sizes were mainly about $20 \mathrm{~nm}$, but ranging from 2-30 nm in different situations. Due to the biocompatibility of starch synthesized silver nanoparticles, this method can be used for medicinal purposes. Both kinds of nanosilver containing starch sources have excellent antibacterial activity.

Conclusions: The current study indicated that silver nanoparticles produced using different starches had strong antibacterial activities on the studied standard and pathogenic bacteria including gram positive and gram negative.
\end{abstract}

Keywords: Silver; nanoparticles; Anti-Bacterial Agents; Green Chemistry Technology

Copyright (c) 2013, Ahvaz Jundishapur University of Medical Sciences; Published by Kowsar Corp.

Article type: Research Article; Received: 14 Apr 2012; Revised: 20 Jun 2012; Accepted: 02 Oct 2012; Epub: 01 Jun 2013 ; Ppub: Jun 2013

Implication for health policy/practice/research/medical education:

This subject is needs to be completed and related to many of the mentioned areas of research, medical education and health policy makers for development of drugs without any complications and or side-effects.

-Please cite this paper as:

Adeli M, Hosainzadegan H, Pakzad I, Zabihi F, Alizadeh M, Karimi F. Preparation of the Silver Nanoparticle Containing Starch Foods and Evaluation of Antimicrobial Activity. Jundishapur J Microbiol. 2013;6(4): e5075. DOI:10.5812/jjm.5075.

Copyright @ 2013, Ahvaz Jundishapur University of Medical Sciences; Published by Kowsar Corp.

This is an Open Access article distributed under the terms of the Creative Commons Attribution License (http://creativecommons.org/licenses/by/3.0), which permits unrestricted use, distribution, and reproduction in any medium, provided the original work is properly cited. 


\section{Background}

Silver is known as one of the elements used from ancient time by human for treatment purposes, and recently is being used in many aspects of human life from health and hospitals to industry. In previous centuries, human beings have used fine and grinded silver for treatment of diseases (1) and nowadays potential applications of metal nanoparticles in the era of nanoscience and technology have generated an explosion in the scientific interest in this class of materials over the last decade (2-8).

One of the interesting aspects of this revolution was the extensive studies of antimicrobial effects of silver nanoparticles which in turn resulted in production of nanosilver-based materials with a potent and broad spectrum of antimicrobial activities (9-12). Upon reaching nanoscale, like other nanomaterials and primarily by virtue of extremely small size, silver particles exhibit remarkably unusual physicochemical properties and biological activities. Great research efforts have been made in this respect and exciting and encouraging results have been obtained (13-15). Silver nanoparticles are emerging as one of the fastest growing products in the nanotechnology industry. It is estimated that among all the nanomaterials in medical and healthcare sector, nanosilver application has the highest degree of commercialization (16).

On the other hand there is an emphasis to synthesize metal nanoparticles using synthetic protocols called "green chemistry" in which using toxic solvents and generating toxic byproducts or waste is eliminated or minimized (17).It is noteworthy that, green chemistry has been introduced to scientific literature by Paul Anastase in 1992. It means continuous search for better and cleaner methods (3). Based on scientific resources green chemistry products must have 3 important characteristics : 1 ) to be environmentally friendly 2) to have good marketing, and 3) to be economical for all social groups $(18,19)$. Hence many research groups in the field of nanoparticle synthesis have turned to biological systems for inspiration.

Based on this strategy the biosynthesis of silver nanoparticles is also being the main aim of many projects of the world in which organisms are used to produce the silver nanoparticles (20-25).

Due to a strong relationship between the size and morphology of nanoparticles and their properties, there is a great interest in synthesizing silver nanoparticles with a controlled size or shape (26-29). Synthesizing silver nanoparticles with a controlled size or shape using "green chemistry" is favored because of the combination of advantages of "green chemistry" and obtaining the metal nanoparticles with well-defined morphology (3032).

Unfortunately, controlling size and shape simultaneously, remains an extremely difficult task, since the outcome of these syntheses is generally determined by a number of factors and is only partially characterized by the interplay of kinetics and thermodynamics. This difficulty increases when silver nanoparticles with a controlled size and shape should be synthesized by "green chemistry.

A simple way to synthesize silver nanoparticles with well-defined size and shape using "green chemistry" is reported here. In this method not only silver nanoparticles are well-defined in the case of size and shape but also they are dispersed in the matrix in a regular order. On the other hand studying the size and shape of nanoparticles are necessary to optimize antimicrobial activities of synthesized nanoparticles.

There are not many researches on antibacterial effects of foods produced by simple chemical methods containing nanosilver.

\section{Objectives}

The current study aimed to evaluate the production of food sources containing nanosilver and to study the antibacterial activity of the mentioned nanofoods.

\section{Materials and Methods}

\subsection{Usual Method for Preparation of Silver Nanoparticles}

In the current study silver nanoparticles were produced using two types of potato starch (named types A and B) and one type of corn starch. But the final results were reported as green potato and Green corn. This process is completely green because reagents of this synthetic protocol are water, potato starch, corn starch and silver nitrate, so according to this study the phrases" green potato" and "green corn" were used to express the products which contained nanosilver.

In this process potato or corn starches were placed in a water solution of silver nitrate in the room temperature overnight, and then they were heated in water at $80^{\circ} \mathrm{C}$ for $2 \mathrm{~h}$. The color of potato and corn starch turned to black during formation of nanoparticles. After reaction they were cooled and washed by distillated water. The diffused silver nitrate to the potato or corn starch was removed using dialysis in deionized water for $12 \mathrm{~h}$.

\subsection{Bacterial Strains}

In the current study one standard bacterium of S. aureus ATCC 25923, and 3 pathogenic bacteria including Klebsiella pneumoniae, Escherichia coli, and Pseudomonas aeruginosa were used. Pathogenic bacteria were isolated from different patient cases and were confirmed as hospital acquired infections by infection control committee of Shohaday Ashayer hospital, Khorram Abad, Iran. All 
of them were resistant against routine antibiotics and K.pneumoniae and P.aeruginosa were pan resistant. All of the bacterial strains were confirmed by standard morphologic and phenotypic tests.

\subsection{Preparation of Green Potato and Green Corn Starches for Antimicrobial Study}

Green potato and green corn were homogenized by sterile sampler tips after addition of $5 \mathrm{ml}$ sterilized normal saline. Supernatants were collected in sterile conditions for antibacterial activity by the following method:

Antibacterial effects (MIC) of supernatants prepared from green potato and green corn were done by micro dilution broth. Eight sterile wells in 8 separate rows of Nonclon micro plates were specified for each of the samples. Two hundred $\mu \mathrm{l}$ of supernatants was added into the first well of 8 rows in micro plate with labeling of four different bacteria. (each supernatant in 4 wells) then 100 $\mu \mathrm{l}$ of freshly prepared Muller - Hinton broth (Difco Laboratories, Detroit, Mich.) was pouredinto the wells second to 8th , after that two fold dilutions were made from first to 7 th well in each row by pipetting and transferring 100 $\mu \mathrm{l}$ from first to 7th wells , and the 8th well served as control. Hundred $\mu \mathrm{l}$ of 0.5 McFarland turbidity suspensions of bacteria freshly prepared from 4 hour cultures in MHB inoculated in two rows (for each of the bacteria two rows were used one for potato and the other for corn suspensions) respectively.

After overnight incubation at $35{ }^{\circ} \mathrm{C}$ all of the inoculated wells were observed exactly for any growth of bacteria. Lowest dilution of supernatants that inhibited growth of microorganisms, was considered as Minimum Inhibitory Concentration (MIC). For further confirmation of the observed results, a sample of wells was sub cultured on Brain Heart Infusion Agar (33). Supernatants of raw heated potato and corn were used as control.

\section{Results}

Figure 1 shows the TEM image of silver nanoparticles produced using potato starch type A. In this Figure silver nanoparticles are well dispersed in the matrix. As indicated, the distribution of their size is narrow and it is around $20 \mathrm{~nm}$. Interestingly the morphology of all nanoparticles is controlled by starch structure and in different regions of the potato starch, shapes of nanoparticles are different. In Figure 1 the shape of nanoparticles is octahedral.

Closer study of TEM image of potato starch (type A) shows that the direction of all nanoparticles is the same and they have a very regular order in the matrix ( Figure 2 ).

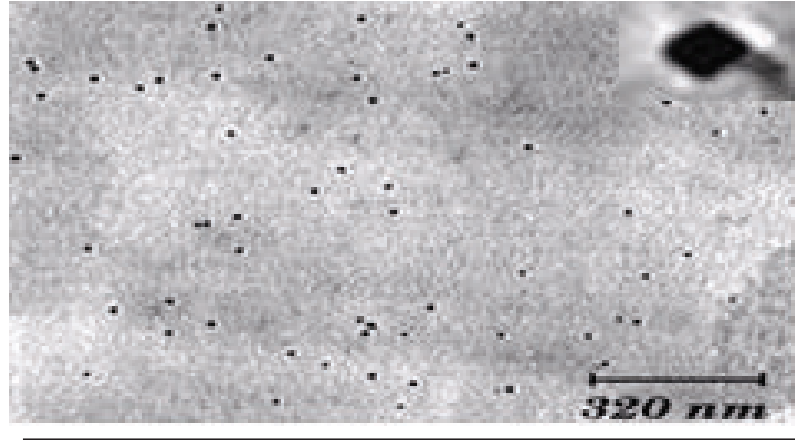

Figure 1 TEM Image of Silver Nanoparticles in the Potato Type A.

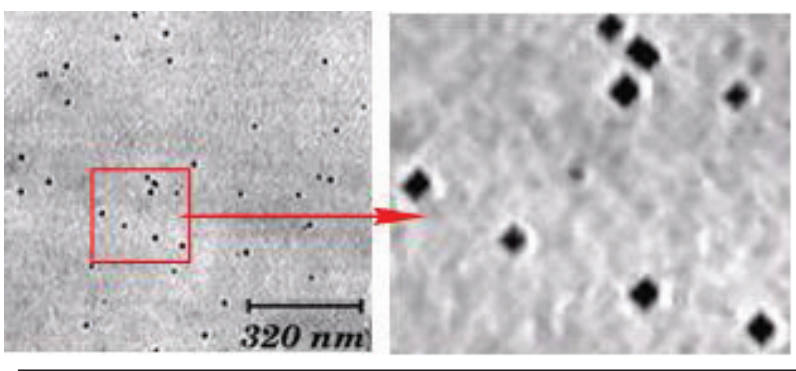

Figure 2. Well Ordered Silver Nanoparticles in Potato Type A.

Figure $3 a$ shows a part of potato starch (type A) with clear borders in which size and shape of nanoparticles are not similar to those of the observed parts in Figures 1 and 2. In Figure $3 b$ which is a TEM image of this part with higher magnification, clearly the shape of nanoparticles is spherical and the size of nanoparticles is between 2-3 $\mathrm{nm}$. Hence different parts of potato starch can produce different nanoparticles with different shape, size and order.

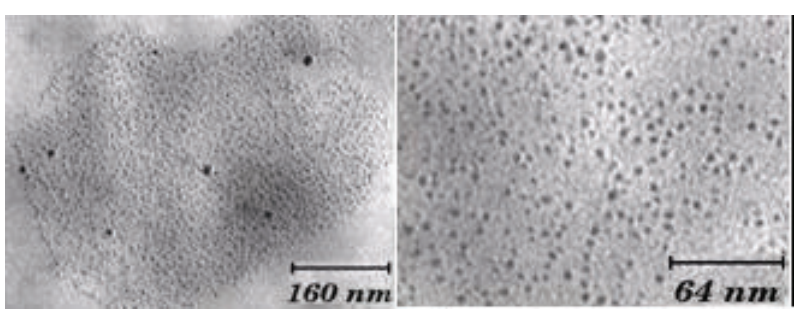

Figure 3. A Part of Potato Type A With Clear Borders in Which Size and Shape of Silver Nanoparticles Are Different From Other Parts.

Extraction of silver nanoparticles from potato was possible using suitable reagents such as mercaptoethanol. Figure 4 displays the UV spectra of aqueous solution of extracted silver nanoparticles. This figure shows a Plasmon absorbance band around $375 \mathrm{~nm}$ for silver nanoparticles. In order to investigate the effect of the type of potato starch on the order, morphology and size of nanopar- 
ticles another type of potato starch (type B) was used to produce silver nanoparticles.

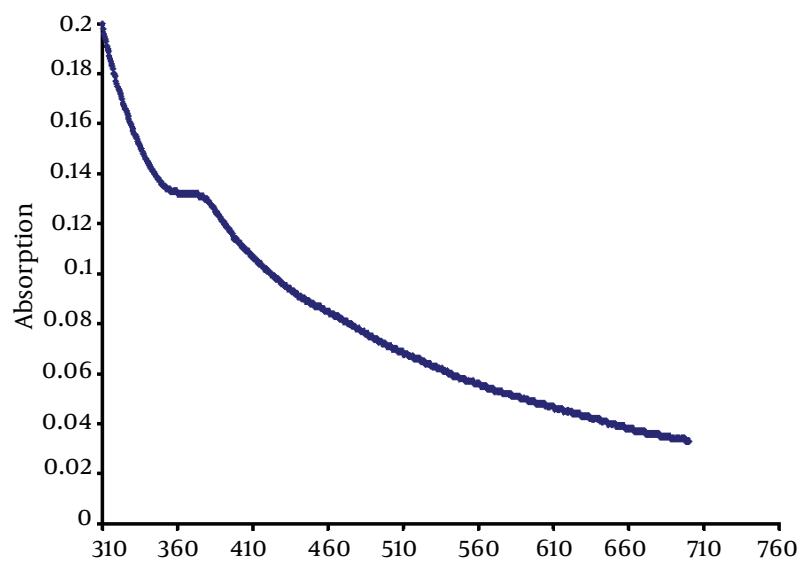

Figure 4. UV-Vis Spectra of Extracted Silver Nanoparticles by Mercaptoethanol.

Figures $5 a$ and $b$ show the TEM image of silver nanoparticles produced using potato starch type B. Here distribution of size is higher than that of type A, nanoparticles are dispersed in the potato matrix irregularly and the shape of nanoparticles in the whole matrix is spherical. Nanoparticles are bigger and their size is between 20$30 \mathrm{~nm}$. In this case again some regions can be observed in which nanoparticles are assembled and their size is smaller than other parts of the potato starch ( Figure $5 b$ ).

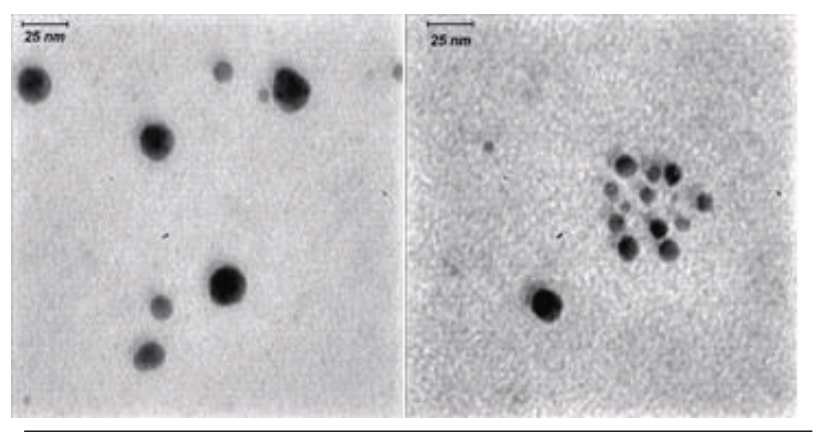

Figure 5. Silver Nanoparticles Synthesized by Potato Type B.

Figure 6 shows TEM image of silver nanoparticles produced using corn starch. In this case the size distribution of nanoparticles is much higher than that of potato starch and several types of nanoparticles in the case of morphology are appeared. In spite of the fact that potato starch in different regions with clear border were not recognized the whole matrix is the same.

As mentioned above, due to the biocompatibility of potato and corn starch, silver nanoparticles in potato and corn starch matrix can be used for clinical purposes. Results of micro dilution study indicated that antibacterial activity of supernatants were identical upon 4 bacterial strains. Filter sterilized ( $0.22 \mu$ Millipore) supernatants of the mentioned green starches did not have any antibac- terial effects on bacteria, probably because of absorption and trapping of silver ions in filter materials.



Figure 6 TEM Image of Silver Nanoparticles Produced Using Corn.

A sample of microdilution plates is indicated in Figure 7. Pyocyanin production of P.aeruginosa was used in rows 3 and 4 as an indicator for observational confirmation of bacterial growth, (green pigments in 6th and 7th wells of 3 and 4 rows respectively), grey turbidity of wells indicates decreasing concentration of Nanosilver, but not bacterial growth in rows.

Figure 7. Growth of Bacteria Indicated in Microplate Serial Dilution, the Pattern of Wells Was as Follow:

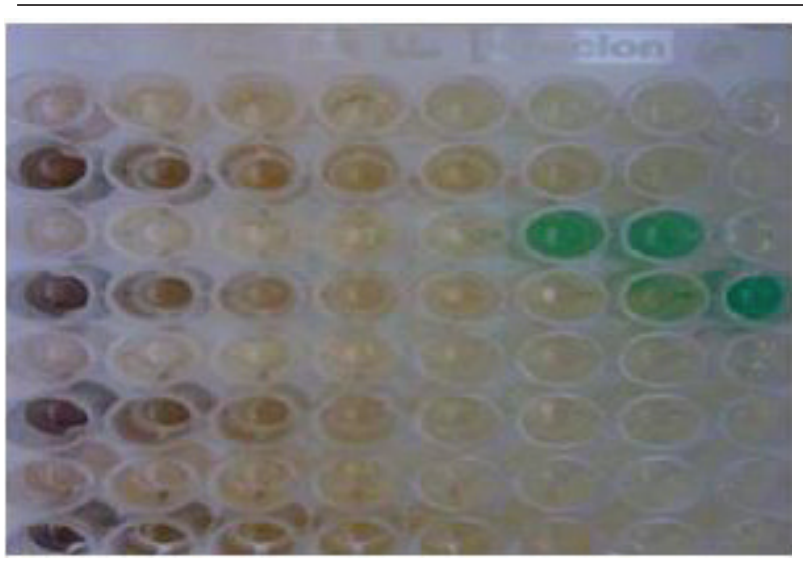

Rows from top of the micro plate to down: 1: S. aureus ATCC 25923 plus potato 2: S. aureus ATCC 25923 plus corn 3: P. aeruginosa plus potato 4: P. aeruginosa plus corn 5: E. coli plus potato 6: E. coli plus corn 7: $k$. pneumoniae plus potato $8: k$. pneumoniae plus corn

\section{Discussion}

Silver nitrate was used as a modifying agent of potato and corn to produce green products in a small scale. Silver is one of the known antimicrobial agents worldwide. This element could be easily converted to nano particles of $10 \mathrm{~nm}$ diameters by nanotechnology that in turn renders it to a potent antimicrobial agent that affects many 
different species of bacteria, including Salmonella typhi, P.aeruginosa, and Mycobacterium tuberculosis and viruses $(11,12,16,34-36)$.

The preliminary studies indicated that silver nanoparticles in potato and corn starch had strong antibacterial activities on the studied bacteria. It is noteworthy that two species including K. pneumoniae and P. aeruginosa were isolated from hospital infections confirmed by National Nosocomial Surveillance System (NNIS) in Shohaday Ashayer hospital, Khorram Abad. This species were also pan-resistant against all of the antibiotic panels in the susceptibility testing, including imipenem.

Petrus etal in their study proposed that Nano Colloidal Silver could be used against food borne antibiotic resistant pathogens as a bacteriostatic agent rather than bactericide.(1)

In a similar study Sathishkumar et al. have produced silver nanoparticles from bark extract and powder of novel Cinnamon zeylanicum that have exerted antimicrobial activity on E. coli. Sathishkumar and colleagues in their study described that size of the produced particles is under the control of $\mathrm{pH}$ (37). Nilda et al. studied the effects of different nanoparticle sizes on MRSA and non -MRSA and indicated that nanosilvers have bactericidal activity on both bacteria, and resistant patterns of the mentioned bacteria are not important in its activity. On the other hand the study of cytotoxicity of different sizes of nanoparticles on Hela cells indicate that particles with lower than $10 \mathrm{~nm}$ diameter have better antibacterial activity and they are also nontoxic to Hela lines. (9)

Although application of nanosilver as particulate or bulk form probably exerts toxic effects on human and mammalian cells, but in nano form they have beneficial effects as a broad spectrum of antimicrobial activity (10). Fidel Martínez-Gutierrez et al. in their study indicated that $24 \mathrm{~nm}$ silver nanoparticles have antibacterial activity against resistant strains of bacteria, and some anti coagulation activity, they also induce inflammatory response in macrophages (38).

The current study findings suggest that green products are economical, non invasive and environmentally friendly materials in combating against infections. In addition, their application needs no skill because it needs only oral consumption. After absorption the nano particle moves through the body and reaches to the site of infection. This encouraging result highlights the need to study its possible side effects in vivo and on human volunteers.

Such kinds of processed foods (containing nanosilver) could be used as immune system booster, in treatment of systemic and latent infections in human and hospital acquired antibiotic resistant infections. in addition, this technique may be used as a suitable method to preserve foods, But in comparison with antibiotics and other antimicrobials application of nanosilver containing products, is very easy (oral as food), cheap, noninvasive, broad spectrum and finally without known resistance problems. In addition such foods are completely environmentally friendly. But comprehensive studies on nanosilver are needed not to have cytotoxicity on humans (in vitro and in vivo) and development of resistance in bacteria, in order to prescribe such products for human.

\section{Acknowledgements}

Authors would like to thank "Iranian Nanotechnology Initiative" to have supported this work financially.

\section{Financial Disclosure}

None declared.

\section{Funding/ Support}

This research was supported financially by "Iranian Nanotechnology Initiative".

\section{Authors' Contribution}

None declared.

\section{References}

1. Petrus EM, Tinakumari S, Chai LC, Ubong A, Tunung R, Elexson $\mathrm{N}$, et al. A study on the minimum inhibitory concentration and minimum bactericidal concentration of Nano Colloidal Silver on food-borne pathogens. Int Food Res J. 2011;18:55-66.

2. Andersson Martin, Alfredsson Viveka, Kjellin Per, Palmqvist Anders EC. Macroscopic Alignment of Silver Nanoparticles in Reverse Hexagonal Liquid Crystalline Templates. Nano Lett 2002;2(12):1403-1407.

3. Jin Rongchao, Cao YunWei, Mirkin Chad A, Kelly KL, Schatz George C, Zheng JG. Photoinduced Conversion of Silver Nanospheres to Nanoprisms. Science. 2001;294(5548):1901-1903.

4. Lee JS, Lytton-Jean AK, Hurst SJ, Mirkin CA. Silver nanoparticleoligonucleotide conjugates based on DNA with triple cyclic disulfide moieties. Nano Lett. 2007;7(7):2112-5.

5. Lin Xue Zhang, Terepka Alexander D, Yang Hong. Synthesis of Silver Nanoparticles in a Continuous Flow Tubular Microreactor. Nano Lett. 2004;4(11):2227-2232.

6. Sun J, Ma D, Zhang H, Liu X, Han X, Bao X, et al. Toward monodispersed silver nanoparticles with unusual thermal stability. J Am Chem Soc. 2006;128(49):15756-64.

7. Ulman A. Formation and Structure of Self-Assembled Monolayers. Chem Rev. 1996;96(4):1533-1554.

8. Zheng J, Ding Y, Tian B, Wang ZL, Zhuang X. Luminescent and Raman active silver nanoparticles with polycrystalline structure. $J$ Am Chem Soc. 2008;130(32):10472-3.

9. Ayala-Nunez NV, Lara Villegas HH, del Carmen Ixtepan Turrent L, Rodríguez Padilla C. Silver nanoparticles toxicity and bactericidal effect against methicillin-resistant staphylococcus aureus: Nanoscale does matter. Nanobiotechnology. 2009;5(1):2-9.

10. Martinez-Gutierrez F, Thi EP, Silverman JM, de Oliveira CC, Svensson SL, Vanden Hoek A, et al. Antibacterial activity, inflammatory response, coagulation and cytotoxicity effects of silver nanoparticles. Nanomedicine. 2012;8(3):328-36.

11. Vedpriya A, Ratika K, Manbir K, Anita G. Silver _nanoparticles as a potent antimicrobial agent: A Review. Pharmacologyonline. 2011;3:118-124.

12. Yang EJ, Jang J, Kim S, Choi IH. Silver Nanoparticles as a Smart Antimicrobial Agent. J Bacteriol Virol. 2012;42(2):177-179.

13. Evanoff DD, Jr, Chumanov G. Synthesis and optical properties of silver nanoparticles and arrays. Chem phys chem. 2005;6(7):122131. 
14. Lee KS, El-Sayed MA. Gold and silver nanoparticles in sensing and imaging: sensitivity of plasmon response to size, shape, and metal composition. J Phys Chem B. 2006;110(39):19220-5.

15. Liu HH, Cao X, Yang Y, Liu MG, Wang YF. Array-based nano-amplification technique was applied in detection of hepatitis E virus.J Biochem Mol Biol. 2006;39(3):247-52.

16. Chen X, Schluesener HJ. Nanosilver: a nanoproduct in medical application. Toxicol Lett. 2008;176(1):1-12.

17. Raveendran P, Fu J, Wallen SL. Completely "green" synthesis and stabilization of metal nanoparticles. J Am Chem Soc 2003;125(46):13940-1.

18. Clark JH. Green chemistry: challenges and opportunities. Green Chem.1999;1(1):1-8.

19. Tundo P, Anastas P, Black DS, Breen J, Collins T, Memoli S, et al. Synthetic pathways and processes in green chemistry. Introductory overview. Pure Appl Chem. 2000;72(7):1207-1230.

20. Ahmad Absar, Mukherjee Priyabrata, Senapati Satyajyoti, Manda Deendayal, Khan MIslam, Kumar Rajiv, et al. Extracellular biosynthesis of silver nanoparticles using the fungus Fusarium oxysporum. Colloids and Surfaces B: Biointer faces. 2003;28(4):313-318.

21. Chandran SP, Chaudhary M, Pasricha R, Ahmad A, Sastry M. Synthesis of gold nanotriangles and silver nanoparticles using Aloe vera plant extract. Biotechnol Prog. 2006;22(2):577-83.

22. Gardea-Torresdey JL, Parsons JG, Gomez E, Peralta-Videa J, Troian HE, Santiago P, et al. Formation and Growth of Au Nanoparticles inside Live Alfalfa Plants. Nano Lett. 2002;2(4):397-401.

23. Mukherjee Priyabrata, Ahmad Absar, Mandal Deendayal, Senapati Satyajyoti, Sainkar Sudhakar R, Khan Mohammad I, et al. Fungus-Mediated Synthesis of Silver Nanoparticles and Their Immobilization in the Mycelial Matrix: A Novel Biological Approach to Nanoparticle Synthesis. Nano Lett. 2001;1(10):515-519.

24. Nair Binoj, Pradeep T. Coalescence of Nanoclusters and Formation of Submicron Crystallites Assisted by Lactobacillus Strains. Crystal Growth \& Design. 2002;2(4):293-298.

25. Sastry M, Ahmad A, Islam Khan M, Kumar R. Biosynthesis of metal nanoparticles using fungi and actinomycete. Curr sci. 2003;85(2):162-170.

26. Callegari A, Tonti D, Chergui M. Photochemically Grown Silver Nanoparticles with Wavelength-Controlled Size and Shape. Nano Lett. 2003;3(11):1565-1568.

27. Chen Y, Munechika K, Ginger DS. Dependence of fluorescence intensity on the spectral overlap between fluorophores and plasmon resonant single silver nanoparticles. Nano Lett. 2007;7(3):690-6.

28. Taguchi Atsushi, Fujii Shintaro, Ichimura Taro, Verma Prabhat, Inouye Yasushi, Kawata Satoshi. Oxygen-assisted shape control in polyol synthesis of silver nanocrystals. Chem Physics Lett 2008;462(1-3):92-95

29. Wiley Benjamin, Herricks Thurston, Sun Yugang, Xia Younan. Polyol Synthesis of Silver Nanoparticles: Use of Chloride and Oxygen to Promote the Formation of Single-Crystal, Truncated Cubes and Tetrahedrons. Nano Lett. 2004;4(9):1733-1739.

30. Joerger R, Klaus T, Granqvist CG. Biologically Produced SilverCarbon Composite Materials for Optically Functional Thin-Film Coatings. Advanced Materials. 2000;12(6):407-409.

31. Klaus T, Joerger R, Olsson E, Granqvist CG. Silver-based crystalline nanoparticles, microbially fabricated. Proc Natl Acad Sci U S A. 1999;96(24):13611-4.

32. Klaus-Joerger T, Joerger R, Olsson E, Granqvist C. Bacteria as workers in the living factory: metal-accumulating bacteria and their potential for materials science. Trends Biotechnol. 2001;19(1):15-20.

33. Methods for Dilution Antimicrobial Susceptibility Tests for Bacteria that Grow Aerobically (currently, CLSI Doc. M7-A9, Clinical Laboratory Standards Institute. [ formerly, National Committee for Clinical Laboratory Standards, or NCCLS]) or anaerobically (CLSI Doc M11-A8).; .

34. Alt V, Bechert T, Steinrucke P, Wagener M, Seidel P, Dingeldein E, et al. [Nanoparticulate silver. A new antimicrobial substance for bone cement]. Orthopade. 2004;33(8):885-92.

35. Song H, Kim Y, Ko K. Antimicrobial mechanisms of silver nanoparticles and their in vivo study for antibiotic alternative. J Kor Inst Met Mater. 2006;44(6):412-417.

36. Song HY, Ko KK, Oh IH, Lee BT. Fabrication of silver nanoparticles and their antimicrobial mechanisms. Eur Cells Mater 2006;11(Suppl 1):58.

37. Sathishkumar M, Sneha K, Won SW, Cho CW, Kim S, Yun YS. Cinnamon zeylanicum bark extract and powder mediated green synthesis of nano-crystalline silver particles and its bactericidal activity. Colloids Surf B Biointerfaces. 2009;73(2):332-8.

38. Shahrokh S, Emtiazi G. Toxicity and Unusual Biological Behavior of Nanosilver on Gram Positive and Negative Bacteria Assayed by Microtiter-Plate. EurJ Biol Sci. 2009;1(3):28-31. 